\title{
Venture capital in academia: does present reality call for more nonprofit venture?
}

\author{
David T. Ting ${ }^{1}$ and Julius J.S. Knowles ${ }^{2}$
}

'Cancer Center, Department of Medicine, Massachusetts Ceneral Hospital, Harvard Medical School, Charlestown, Massachusetts, USA. ${ }^{2}$ Mass General Brigham Innovation Fund, Mass Ceneral Brigham, Cambridge, Massachusetts, USA.

\section{Introduction}

For the past 40 years in biotech, venture capitalists have visited academic medical centers, invested in ideas that taxpayers have funded, created new companies (newcos), and made handsome financial gains from these investments. This investment has led to a boom in the creation of newcos, and wonderful therapies have emerged as a result. Unfortunately, this shift in funding has not benefitted the academic medical center as much, with young talent pulled away from the academic world into biotech. In essence, funds have flowed from the government to venture capitalists to pharmaceutical companies. But we have an opportunity that is even more important, given the clear need to support hospitals and future health care investments, to leverage those ideas to shore up hospital balance sheets, and to fund future medical care. This is not to say that the model is broken - far from it. It is only that a moderate reallocation of funding from venture capitalists to the inventors of those ideas can create a virtuous circle and further improve the ecosystem.

\section{The academic dream: bench to bedside}

The legend of an academic researcher toiling away in an ivory tower is still very much alive. Many physicians and biomedical scientists are driven to find new methods to diagnose and treat human disease, with the ultimate dream of taking something discovered at the bench to the bedside. This is the core mission of the academic research hospital and an engine for bio- medical innovation. A physician-scientist may spend a significant amount of time poring over tissue samples, understanding gene and protein expression patterns, and teasing out potential drug targets, often by themselves or with a small team of graduate students, postdocs, and academic collaborators. Optimally, the effort leads to identification of a drug target and occasionally to a research-grade antibody or small molecule, with variable efficacy in relevant molecular and cellular assays and uncertain value for drug discovery.

At this stage, industry veterans should take on the herculean task of creating a drug development candidate. Academic researchers will look for venture funding to support a startup company outside of their institution. Although the science is exciting with great potential, researchers typically embark on a quixotic series of meetings with lots of questions and no commitment to bring the science forward. The lack of investor interest may be justified, as many of these ideas are speculative and high risk. The financing process leads to significant time lost for scientists, who are pitching ideas instead of doing what they do best - scientific discovery. Even when venture capitalists show interest, scientific founders find themselves in a weak negotiating position, and the wear of selling an idea over many months to years leads to venture capitalists gaining most of the ownership stake.

\section{Mass General Brigham}

At Mass General Brigham (formerly Partners HealthCare), we have 6200 principal

\footnotetext{
Conflict of interest: DTT has received consulting fees from ROME Therapeutics, Merrimack Pharmaceuticals, Ventana Roche, Foundation Medicine, and MilliporeSigma. DTT is a founder of and holds equity in ROME Therapeutics, PanTher Therapeutics, and TellBio, Inc. DTT receives research support from ACD Bio-Techne, PureTech Health LLC, and Ribon Therapeutics. DTT's interests were reviewed and are managed by Massachusetts General Hospital and Partners HealthCare in accordance with their conflict-of-interest policies. JJSK is on the board of Abcuro, Apres Therapeutics, Keros Therapeutics, ROME Therapeutics, and SwanBio Therapeutics and represents the fund on the boards of Akouos, Lysosomal Therapeutics, and Triplet Therapeutics. JJSK has had previous investments in Editas Medicine, Denali Therapeutics, Tilos Therapeutics, and Translate Bio.

Copyright: @ 2020, American Society for Clinical Investigation.

Reference information: / Clin Invest. 2020;130(7):3336-3338. https://doi.org/10.1172/JCI138642.
}

investigators (PIs), who are active in therapeutic areas ranging from neuroscience to oncology to rare diseases and employ technologies, including CRISPR, gene therapy, CAR-T cells, therapeutic oligonucleotides, antibodies, and small molecules. Annually, the technology transfer office receives over 500 PI-submitted invention disclosures (Figure 1), many of which are highly valuable but not the foundation for a strong biotech company. These inventions include diagnostic biomarkers, algorithms, research tools, and surgical devices. It is less common (only about 50 per year) for disclosed concepts to enable the creation of a new company (newco). Mass General Brigham looks for ways to transform these commercializable concepts and turn them into newcos, an example of which was started in 2008 and is discussed below.

\section{Partners Innovation Fund}

In 2008, hospital leaders recognized that it would be financially and strategically beneficial to take propositions created at the hospital into the clinic and then partner with pharmaceutical companies, as opposed to engaging pharma at the scientific hypothesis stage, resulting in potential shelving of ideas when pharma strategy changes. Leaders also recognized that biotech companies have a strong track record of recruiting the relevant drug discovery experts. As a result, the Partners Innovation Fund (PIF) - a venture fund initially with $\$ 35 \mathrm{M}$ under management that has now grown to $\$ 170 \mathrm{M}$ - was created. The original funding came from Massachusetts General Hospital (MGH) and Brigham and Women's Hospital (BWH), with \$17.5M from each hospital. From 2008 to 2014, PIF invested in three to five companies per year but had not yet had financial exits through an acquisition or an IPO, which are the lifeblood of the venture industry. Patient leadership from the hospitals and strong management from the founding 


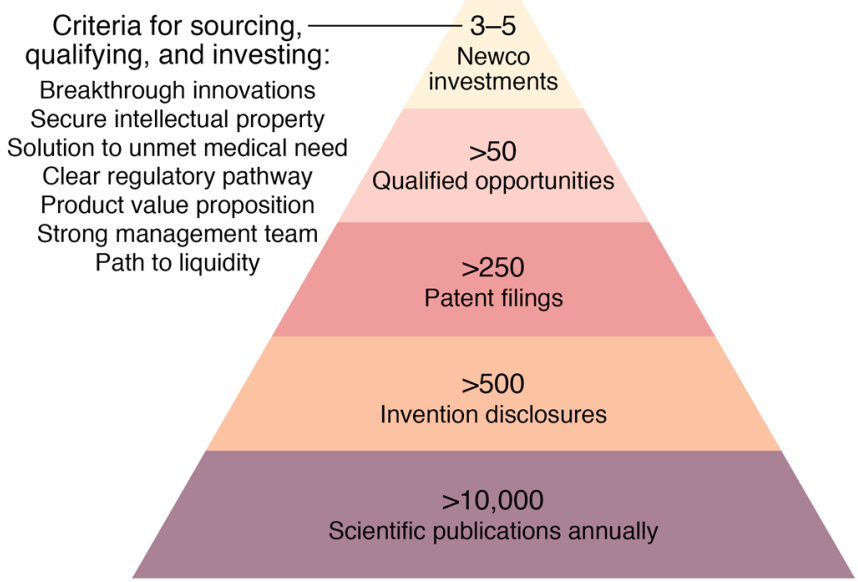

Figure 1. PIF pipeline for next-generation therapeutics. PIF originated from the combined investment of MGH and BWH. Funds are used to invest in institutional PIs at the seed stage to support the development of research concepts and follow through on investment in lead productions for further development as a newco or pharma. A dynamic pipeline of publications, inventions, and PIF investment review produces newcos with a sustainable future.

partners, Roger Kitterman and Carl Berke, kept PIF in the game as seed-stage investments matured. From 2014 through the end of 2019, PIF had 12 exits and achieved top-quartile life science venture returns.

Since 2008, PIF has operated as a typical fund, including Monday afternoon deal meetings with partners and venture associates. PIF now has four partners who are industry veterans and a senior venture associate with a scientific/consulting background. PIF also has an investment committee of leaders in the life science venture and pharmaceutical industries, who must approve investment decisions recommended by the partner team; the investment committee helps maintain objectivity and independence from the host institutions. The fund was designed to maintain adequate reserves for follow-on investment, rather than investing only at the beginning of a company's life and hoping for the best. This design enabled a more rigorous process, as opposed to the previous academic model of making an upfront investment without the ability to influence the company's long-term success. PIF forged a strong connection with the licensing office to identify new opportunities that emerged from our large academic ecosystem. The hospitals also amended the standard licensing agreements to include a term providing PIF with the right to invest in up to $10 \%$ of the seed and subsequent financing rounds. This term enables a more robust investment, with board rights to protect the interests of our investigators and the institution.

\section{Lessons learned}

PIF has helped launch 43 companies in the past 12 years, and several core themes have process. First, ensuring appropriate founding equity to our scientists has been critical for the initiation and continued involvement of scientific founders to propel companies toward success. Scientific founders have worked for many years in the research area, and their insight into the biology or technology is necessary to recruit other venture investors and eventually create a bridge between the academic science and the company team. Second, most academic science is immature as a product, and for sufficient interest from early investors. This concept is sometimes difficult for founders to accept, given that their perception of the potential future value of their idea does not translate to the existing value of the technology. Third, reasonable and timely technology transfer agreements are essential for newcos. Almost all of the company's money at the early stages must go to investing in the company rather than back to the institution, which will provide the company with a sustainable intellectual property portfolio strategy. PIF will ensure that the hospital receives small royemerged as a critical part of the founding therefore low pre-money valuations allow alties so that the long-term upside can be shared. Patent costs must be reimbursed as well as small clinical and commercial milestones. The inherent benefit of having PIF interact with the MGB technology transfer office allows this process to move efficiently, which saves precious time and effort that the company can use to focus on advancing the scientific mission. Last, the choice of a CEO is one of the most important decisions for the success of any newco. This will usually be someone with a deep scientific background in the area of interest and translational expertise to bring a drug or technology into the clinic. This person should also have experience raising funds from investors and the ability to operationalize the team. It is often difficult to find someone who can do it all. In addition, the CEO needs to ensure communication between founders, investors, and the team. The balance is tricky and requires great patience from founders and investors, and PIF can serve as an intermediary to help bridge these concepts from the lab to the newco.

\section{Discussion}

In 2017, Mass General Brigham underscored the value of a venture fund that provides a path to develop technologies to advance medicine and serves as an investment that generates significant returns to support the hospital's core mission of clinical care delivery. MGH and BWH invested $\$ 100 \mathrm{M}$ over ten years, and PIF raised $\$ 71 \mathrm{M}$ from external limited partners, based on the strength of returns to date (and derived from the great technologies created at Mass General Brigham). The external funding helped to validate the original model and enriched PIF decision making, as some of the external investors in PIF are pharmaceutical companies, and their scientists act as a sounding board for new investment ideas. With the larger fund, PIF moved from investing $\$ 2-\$ 4 \mathrm{M}$ per company to investing $\$ 6-\$ 10 \mathrm{M}$ per company. Since biotech companies generally need $\$ 50-\$ 100 \mathrm{M}$ to reach an exit, $\$ 6-\$ 10 \mathrm{M}$ allows PIF to have more leverage in the company creation process and can enable a voting board seat, thus providing more influence and oversight on the investment.

One emergent question speaks to the appropriate size of PIF. PIF was clearly not 
large enough at $\$ 35 \mathrm{M}$, but would it be better to be bigger? If PIF were a $\$ 300 \mathrm{M}$ fund, the number of companies invested in could grow to 30 , with $\$ 10 \mathrm{M}$ per newco, or larger investments could be available for projects that need substantially more seed money. A larger fund would bring the scale of technology development needed to retain scientific talent and further leverage the hospital's impact on the local biotech ecosystem.

New venture firms such as PIF are a helpful step in transferring technology out of academia. Nonetheless, many ideas are not yet ready for PIF to tackle. Mass General Brigham has recently added a seed fund to further enable new opportunities called the Translational Innovation Fund (TIF). TIF plans to spend $\$ 5 \mathrm{~K}$ to $\$ 2 \mathrm{M}$ on developing ideas to enable venture syndication. This pre-seed funding, again shepherded by industry veterans, should keep early technology development within the institution and allow for flexibility, especially as early science tends to evolve rapidly. This model should allow the investigator to spend time developing critical assays to de-risk the science for eventual transfer to a newco or pharma. This pre-seed funding not only protects innovative ideas from being discarded too early but also provides better returns for the hospital, either through increased pre-money valuations or via direct licensing to pharma companies, with greater upfront and downstream payments. The TIF model has only recently been added - it is the next phase of experimentation in our academic venture community.

In summary, the expansion of academic ventures will inevitably lead to faster translation of innovation from the bench to the bedside. The use of internal funds to expand on cutting-edge, but still immature, technologies can bring more advances into the clinical realm and provide a financial strategy to feed this engine. Moreover, academic hospitals can decide to use financial gains from innovation to support clinical operations in times of medical crises, such as the one we are facing with COVID-19. Wouldn't it be remarkable to deploy profits from academic innovations to provide the best and most advanced care for our patients instead of for financial gain? Although there will be new challenges on the horizon, the partnership between academic scientists and venture capitalists can expand our ability to increase the speed and number of inventions and continue to transform the landscape of biomedicine.

\section{Acknowledgments}

We acknowledge the feedback from Daniel A. Haber and the PIF team (Roger Kitterman, Carl Berke, Stacey Brenner, Meredith Fisher, and Rosy $\mathrm{Hu}$ ).

Address correspondence to: David T. Ting, Massachusetts General Hospital Cancer Center, Building 149, Thirteenth Street, Room 6-618B, Charlestown, Massachusetts 02129, USA. Phone: 617.240.9402; Email: dting1@mgh.harvard.org. Or to: Julius J.S. Knowles, Partners Innovation Fund, 215 First Street, Suite 500, Cambridge, Massachusetts 02142, USA. Phone: 857.307.2436; Email: jjknowles@ partners.org. 